\title{
FRIABLE VALUES OF PIATETSKI-SHAPIRO SEQUENCES
}

\author{
YILDIRIM AKBAL \\ (Communicated by Kathrin Bringmann)
}

Abstract. We give various estimates for friable values of Piatetski-Shapiro sequences.

\section{INTRODUCTION}

For any positive integer $n$, let $P^{+}(n)$ be the largest prime factor of $n$ with the convention that $P^{+}(1)=0$. For any $y \geqslant 2$, an integer $n$ is called $y$-friable whenever $P^{+}(n) \leqslant y$. Friable numbers show up in diverse areas of number theory; in particular, for any non-zero polynomial $f \in \mathbb{Z}[x]$, the quantity

$$
\Psi_{f}(x, y)=\#\left\{n \leqslant x: P^{+}(|f(n)|) \leqslant y\right\}
$$

plays an important role in modern factorization algorithms. Apart from the linear cases, our current knowledge of $\Psi_{f}$ is limited unless $y$ is not large. To be more explicit, Dartyge et al. (cf. [4]) showed that when $f$ is a product of irreducible polynomials of degree $d_{i}, i=1, \ldots, k$, then $\Psi_{f}(x, y)$ is of order $x$ for $y \geqslant x^{d-1 / l+\varepsilon}$ where $d=\max _{i=1, \ldots, k}\left\{d_{i}\right\}$ and $l$ is the number of factors having degree $d$.

Moreover, with the same notation as above, Greg Martin (cf. [12]) proved under very strong assumptions that one has

$$
\Psi_{f}(x, y) \sim \rho\left(d_{1} u\right) \rho\left(d_{2} u\right) \ldots \rho\left(d_{k} u\right) x \quad(x \rightarrow \infty)
$$

for the same range of $y$, where $u=\log x / \log y$ and $\rho(u)$ is the Dickman function (see e.g. [16, §III.5]). It is, however, expected that (1.1) should hold for any $y$ greater than any fixed power of $x$. For further information we refer the reader to [7] (see also [1]).

Motivated by the problem above, we shall exhibit non-linear sequences for which an analogue of (1.1) holds. To this end, for non-integer $c>1$, we study friable values of Piatetski-Shapiro sequences defined by $\overline{\left.\left\lfloor n^{c}\right\rfloor\right\}_{n \in \mathbb{N}}}$, where $\lfloor x\rfloor$ is the floor of $x$. Piatetski-Shapiro was the first to prove a "prime number theorem" for these sequences when $1<c<12 / 11$ (see [13,14]).

For $c \geqslant 1$, we let

$$
\Psi_{c}(x, y)=\#\left\{n \leqslant x: P^{+}\left(\left\lfloor n^{c}\right\rfloor\right) \leqslant y\right\}
$$

Received by the editors April 11, 2016 and, in revised form, November 24, 2016.

2010 Mathematics Subject Classification. Primary 11N32; Secondary 11L03, 11B83.

Key words and phrases. Exponential sums over friable numbers, sparse sequences.

This work was supported by the Scientific and Technological Research Council of Turkey (114F404). We thank the referee for carefully reading the manuscript. 
Here it is worth mentioning that a similar set was studied by Baker et al. 3, Theorem 6], and their result implies that

$$
\Psi_{c}\left(x, x^{\varepsilon}\right) \gg x^{1-\varepsilon} \text { for every } 1<c<24979 / 20803 .
$$

In the case that $c=1$ (that is, the case $f(x)=x$ ), Hildebrand (cf. [10]) showed that for any fixed $\varepsilon>0$,

$$
\Psi_{1}(x, y)=x \rho(u)\left\{1+O\left(\frac{\log (u+1)}{\log y}\right)\right\}
$$

holds uniformly for $\exp \left((\log \log x)^{5 / 3+\varepsilon}\right) \leqslant y \leqslant x$ whenever $x \geqslant x(\varepsilon)$. He also established the less precise result

$$
\Psi_{1}(x, y)=x \rho(u) \exp \left(O\left(u \exp (-\log u)^{3 / 5-o(1)}\right)\right)
$$

holding uniformly in the wider range $\log ^{1+\varepsilon} x \leqslant y \leqslant x$ whenever $x \geqslant x(\varepsilon)$.

In our setting, we first show that an analogue of the expected asymptotic (1.1) is true if $1<c<1669 / 1389=1.20158 \ldots$, uniformly for a range of $y$ depending only on $c$.

Theorem 1.1. For any fixed $1<c<1669 / 1389$, and for any $\varepsilon>0$ fixed, there is a number $\kappa>0$ such that for any fixed $0<\eta<\kappa$, and $x$ large depending only on $c$ and $\varepsilon>0$, the asymptotic formula

$$
\Psi_{c}(x, y)=x \rho(c u)\left\{1+O\left(\frac{\log (c u+1)}{\log y}\right)\right\}
$$

holds uniformly for $y$ in the range

$$
\exp \left(\left(\log \log x^{c}\right)^{5 / 3+\varepsilon}\right) \leqslant y \leqslant \mathcal{F}(x, c, \eta)
$$

where

$$
\mathcal{F}(x, c, \eta)=\left\{\begin{aligned}
\frac{x}{\log ^{18} x} & \text { if } 1<c<2509 / 2229, \\
x^{\frac{1669-1389 c}{280}-\eta} & \text { if } 2509 / 2229 \leqslant c<1669 / 1389 .
\end{aligned}\right.
$$

Here we note that following the proof of Theorem 1.1 one may decrease the power of $\log$ in $\mathcal{F}(x, c, \eta)$ above which will not be pursued here for the sake of brevity.

The next theorem indicates that at the cost of losing the asymptotic in Theorem 1.1, one is able to extend the range of $y$.

Theorem 1.2. Let $\varepsilon>0$ be small and fixed. Let $1<c<1669 / 1389$ be fixed. Then there is a real number $\kappa>0$ such that for any fixed $0<\eta<\kappa$, and x large depending only on $c$ and $\varepsilon$, one has

$$
\Psi_{c}(x, y)=x \rho(c u) \exp \left(O\left(c u \exp \left(-(\log c u)^{3 / 5-o(1)}\right)\right)\right)
$$

uniformly for

$$
\log ^{4128 c /(1669-1389 c)+\varepsilon} x \leqslant y \leqslant \mathcal{F}(x, c, \eta)
$$

where $\mathcal{F}(x, c, \eta)$ is defined in Theorem 1.1 .

The proofs of Theorem 1.1 and Theorem 1.2 rely on the factorization property of friable numbers given in Lemma 1.6, creating exponential sums in (1.15). The methods to deal with these exponential sums differ depending on the size of $y$; that is, when $y$ is small, Lemma 1.13 is sufficient, while for larger values of $y$, we take into account the variation of the related parameters by combining Lemmata 1.13 
and 1.14. At this point, it is worth mentioning that if one assumes the exponent pair hypothesis, then Lemma 1.13 reveals that the range of $c$ in Theorems 1.1 and 1.2 widens up to $1<c<1.25$ whenever $y$ is not large.

\subsection{Preliminaries and notation.}

1.1.1. Notation. Given a real number $x$, we write $e(x)=e^{2 \pi i x},\{x\}$ for the fractional part of $x$ and $\lfloor x\rfloor$ for the greatest integer not exceeding $x$.

The notation $\|x\|$ is used to denote the distance from the real number $x$ to the nearest integer. For $N \geqslant 1$ integer, we write $n \sim N$ to mean that $n$ lies in an interval contained in $(N, 2 N]$, whenever it is clear from content, otherwise we shall denote it specifically. We also put $\delta=1 / c$.

We recall that for functions $F$ and real non-negative $G$, the notation $F \ll G$ and $F=O(G)$ are equivalent to the statement that the inequality $|F| \leqslant \alpha G$ holds for some constant $\alpha>0$. Further we use $F \asymp G$ to indicate that both $F \gg G$ and $F \ll G$ hold. For any integer $n \geqslant 1$, we define $P^{-}(n)$ to be the smallest prime factor of $n$ with the convention that $P^{-}(1)=-\infty$.

We put

$$
\psi(x)=x-\lfloor x\rfloor-1 / 2, \quad \text { and } \quad \Delta \psi(x)=\psi\left(-(x+1)^{\delta}\right)-\psi\left(-x^{\delta}\right) .
$$

Throughout this paper we reserve the pairs $(k, l)$ to be exponent pairs for which the reader is referred to [6, §3].

In a slight departure from convention, we shall frequently use $\varepsilon$ to mean a small positive number possibly not the same at each occurence.

1.1.2. Preliminaries. In this section we state several lemmata in order to reduce the proof of Theorems 1.1 and 1.2 to exponential sums.

Lemma 1.3. Suppose $c>1$, and $1<y \leqslant x^{c}$; then

$$
\Psi_{c}(x, y)=\sum_{\substack{n \leqslant x^{c} \\ P^{+}(n) \leqslant y}} \delta n^{\delta-1}+\sum_{\substack{n \leqslant x^{c} \\ P^{+}(n) \leqslant y}} \Delta \psi(n)+O(1)
$$

where the implied constant depends only on $c$.

Proof. The equality $m=\left\lfloor n^{c}\right\rfloor$ holds precisely when $m \leqslant n^{c}<m+1$, or equivalently, when $-(m+1)^{\delta}<-n \leqslant-m^{\delta}$. Hence,

$$
\sum_{\substack{n \leqslant x \\ P^{+}\left(\left\lfloor n^{c}\right\rfloor\right) \leqslant y}} 1=\sum_{\substack{m \leqslant x^{c} \\ P^{+}(m) \leqslant y}}\left(\left\lfloor-m^{\delta}\right\rfloor-\left\lfloor-(m+1)^{\delta}\right\rfloor\right)+O(1) .
$$

The desired result follows upon recalling the facts

$$
(m+1)^{\delta}-m^{\delta}=\delta m^{\delta-1}+O\left(m^{\delta-2}\right)
$$

and

$$
\sum_{\substack{m \leqslant x^{c} \\ P^{+}(m) \leqslant y}} m^{\delta-2}=O(1)
$$

To deal with the penultimate term on the right hand side of (1.5), we use the following result. 
Lemma 1.4. Suppose $1 \leqslant z<y$. Let $N \geqslant 1$, and let $N<N^{\prime} \leqslant 2 N$. Suppose $1 \leqslant H_{N}<\infty$ is a real number depending on $N$. Then

$$
\begin{aligned}
\sum_{\substack{N<n \leqslant N^{\prime} \\
z<P^{+}(n) \leqslant y}} \Delta \psi(n) \ll N H_{N}^{-1}+H_{N}^{1 / 2} N^{\delta / 2}+H_{N}^{-1} N^{1-\delta} \log H_{N} \\
+N^{\delta-1} \max _{N<N^{\prime \prime} \leqslant 2 N} \sum_{1 \leqslant h \leqslant H_{N}}\left|\sum_{\substack{N<n \leqslant N^{\prime \prime} \\
z<P^{+}(n) \leqslant y}} e\left(h n^{\delta}\right)\right|
\end{aligned}
$$

where the implied constant depends only on $\delta$.

Proof. The proof follows using standard arguments; see e.g. [6, §4].

Lemma 1.5. For $H_{2} \geqslant H \geqslant H_{1} \geqslant 1$, let

$$
L(H)=\sum_{i=1}^{m} A_{i} H^{a_{i}}+\sum_{j=1}^{n} B_{j} H^{-b_{j}}
$$

where $A_{i}, B_{j}, a_{i}$ and $b_{j}$ are positive real numbers. Then

$$
\max _{H_{1} \leqslant H \leqslant H_{2}} L(H) \ll \sum_{i=1}^{m} A_{i} H_{1}^{a_{i}}+\sum_{j=1}^{n} B_{j} H_{2}^{-b_{j}}+\sum_{i=1}^{m} \sum_{j=1}^{n}\left(A_{i}^{b_{j}} B_{j}^{a_{i}}\right)^{1 /\left(a_{i}+b_{j}\right)}
$$

where the implied constants depend only on $m$ and $n$.

Proof. See [6, Lemma 2.4.].

The following lemma allows one to factorize friable numbers in a convenient manner.

Lemma 1.6. Suppose that $2 \leqslant y \leqslant R \leqslant n \leqslant x$, with $P^{+}(n) \leqslant y$. Then there is a unique triple $(p, u, v)$ satisfying,

(i) $n=p u v$,

(ii) $p \leqslant y$,

(iii) $R / p<v \leqslant R$ with $P^{-}(v) \geqslant p$ and $P^{+}(v) \leqslant y$,

(iv) $u \leqslant x / p v$ with $P^{+}(u) \leqslant p$.

Proof. See e.g. [17, Lemma 10.1].

1.1.3. Exponential sums with monomials. For positive integers $H, K$ and $L$, a real number $X>0 ; a(h, n)$ complex numbers with norm at most one, and $b(m)$ complex numbers satisfying $\sum_{L<m \leqslant 2 L}|b(m)|^{2} \ll L \log ^{2 A} L$ for some $A \in \mathbb{R} ; \alpha, \beta$ and $\gamma$ real numbers, we define

$$
S:=\sum_{H<h \leqslant 2 H} \sum_{K<n \leqslant 2 K} a(h, n) \sum_{L<m \leqslant 2 L} b(m) e\left(X \frac{h^{\beta} n^{\gamma} m^{\alpha}}{H^{\beta} K^{\gamma} L^{\alpha}}\right) .
$$

The following theorem is a well-known result of Robert and Sargos.

Lemma 1.7. Suppose $b(m)$ is at most one for all $m \in(L, 2 L]$. Let $\alpha(\alpha-1) \beta \gamma \neq 0$. Then for every $\varepsilon>0$,

$$
S \ll(H K L)^{1+\varepsilon}\left\{\left(\frac{X}{H K L^{2}}\right)^{1 / 4}+\frac{1}{(H K)^{1 / 4}}+\frac{1}{L^{1 / 2}}+\frac{1}{X^{1 / 2}}\right\},
$$

where the implied constant depends only on $\alpha, \beta, \gamma$ and $\varepsilon>0$. 
Proof. See [15, Theorem 1].

The next result is a generalization of the method of estimating so-called Type II sums in [9]. This method was later generalized by Roger Baker (see e.g. [2]) to arbitrary exponent pairs under the assumption $X \geqslant H K$. Here we reprove his theorem in a slightly general manner.

Lemma 1.8. Let $(k, l)$ be an exponent pair. Suppose $\alpha<1$, and $\alpha \beta \gamma \neq 0$. Then

$$
\begin{aligned}
S \ll H L K \log ^{A+1}(2 H K L)\left\{\left(\frac{X^{k}}{L^{1+k-l} H^{k} K^{k}}\right)^{1 / 2(k+1)}+\right. & \frac{1}{(H K)^{1 / 2}}+\frac{1}{X^{1 / 2}} \\
& \left.+\frac{1}{L^{(1+k-l) / 2(k+1)}}\right\}
\end{aligned}
$$

where the implied constant depends only on $\alpha, \beta, \gamma$ and $(k, l)$.

Proof. We may normalize $b(m)$ by $\log ^{A} L$, thus assume that $\sum_{L<m \leqslant 2 L}|b(m)|^{2} \ll$ $L \log ^{2 A} L$. We may also assume $X \geqslant 3$ and $\frac{X^{k}}{L^{1+k-l} H^{k} K^{k}}<1$, otherwise, in either case, the result is trivial. By the Cauchy-Schwarz inequality one has

$$
S^{2} \ll L \sum_{L<m \leqslant 2 L}\left|\sum_{H<h \leqslant 2 H} \sum_{K<n \leqslant 2 K} a(h, n) e\left(X \frac{h^{\beta} n^{\gamma} m^{\alpha}}{H^{\beta} K^{\gamma} L^{\alpha}}\right)\right|^{2} .
$$

We next apply [14, Lemma 5] with $x_{i}=\frac{h^{\beta} n^{\gamma}}{2^{\beta+\gamma+1} H^{\beta} K^{\gamma}}$ and $z_{i}=a(h, n) e\left(X \frac{h^{\beta} n^{\gamma} m^{\alpha}}{H^{\beta} K^{\gamma} L^{\alpha}}\right)$ yielding

$$
S^{2} \ll \frac{L}{\eta} \sum_{\substack{h_{1}, h_{2} \sim H \\ n_{1}, n_{2} \sim K \\|\Delta|<\eta}}\left|\sum_{m \sim L} e\left(2^{\beta+\gamma+1} X \Delta \frac{m^{\alpha}}{L^{\alpha}}\right)\right|
$$

where $\Delta=\frac{h_{1}^{\beta} n_{1}^{\gamma}-h_{2}^{\beta} n_{2}^{\gamma}}{2^{\beta+\gamma+1} H^{\beta} N^{\gamma}}$, and $1 / 2>\eta>0$ to be determined later. We now suppose $1 / 2>\eta \geqslant 1 / X$ and estimate the innermost sum trivially by $L$ whenever $|\Delta|<1 / X$, which by [5, Lemma 1] gives rise to a contribution

$$
\ll \mathcal{E}:=\log (2 H K)\left\{\frac{L^{2} H K}{\eta}+\frac{H^{2} K^{2} L^{2}}{\eta X}\right\} .
$$

Applying the exponent pair $(k, l)$ whenever $\frac{1}{X} \leqslant|\Delta|<\eta$, it follows that

$$
S^{2} \ll \frac{L}{\eta} \sum_{\substack{h_{1}, h_{2} \sim H \\ n_{1}, n_{2} \sim K \\ \frac{1}{X} \leqslant|\Delta|<\eta}}\left\{X^{k}|\Delta|^{k} L^{l-k}+\frac{L}{X|\Delta|}\right\}+\mathcal{E} \ll \mathcal{I}_{1}+\mathcal{I}_{2}+\mathcal{E}
$$

where $\mathcal{I}_{1}$ and $\mathcal{I}_{2}$ denote the contribution of the first and second terms in braces respectively. It is not too hard to see that splitting up the interval $\frac{1}{X} \leqslant|\Delta|<\eta$ into $\ll \log X \ll \log (2 H K L)$ dyadic intervals and applying [5, Lemma 1] on each such interval, one has $\mathcal{I}_{2} \ll \log (2 H K L) \mathcal{E}$. Furthermore, by [5] Lemma 1], it follows that

$$
\mathcal{I}_{1} \log ^{-1}(2 H K) \ll \eta^{k-1} L^{1+l-k} X^{k} H K+\eta^{k} L^{1+l-k} X^{k} H^{2} K^{2} .
$$


Choosing $\frac{1}{X} \leqslant \eta<1 / 2$ optimally by Lemma 1.5, one gets

$$
\begin{aligned}
S^{2} \log ^{-2}(2 H K L) & \ll L^{2} H K+\frac{H^{2} K^{2} L^{2}}{X}+L^{1+l-k} X^{k} H K+L^{1+l-k} H^{2} K^{2} \\
+L^{\frac{1+l+k}{1+k}} & H^{\frac{2+k}{1+k}} K^{\frac{2+k}{1+k}} X^{\frac{k}{1+k}}+H^{2} K^{2} L^{\frac{1+l+k}{1+k}}+L^{1+l-k} X^{k} H^{2-k} K^{2-k}
\end{aligned}
$$

hence the claimed result using the assumptions that were made in the beginning.

The following lemma is not used in the proofs of Theorem 1.1 and Theorem 1.2, since in our case we can bypass it, but is of particular importance because it combines various methods, and, as far as we are aware, does not appear in the literature.

Lemma 1.9. Let $\alpha \theta \beta \gamma(\alpha-1)(\theta-1) \neq 0$. Suppose $K, L, H$ and $P$ are all positive integers. Let

$$
S^{\prime}:=\sum_{\substack{K<n \leqslant 2 K \\ H<h \leqslant 2 H \\ P<r \leqslant 2 P}} a(n, h, r) \sum_{L<m \leqslant 2 L} q(m) e\left(X \frac{h^{\beta} n^{\gamma} m^{\alpha} r^{\theta}}{H^{\beta} K^{\gamma} L^{\alpha} P^{\theta}}\right),
$$

where $a(n, h, r)$ and $q(m)$ are complex numbers with norm at most one. If $(k, l)$ is an exponent pair, then

$$
\begin{aligned}
S^{\prime} \ll H K L P \log ^{3 / 2}( & 2 H K L P)\left\{\frac{1}{X^{1 / 2}}+\frac{1}{(H K)^{1 / 2}}+\frac{1}{L^{1 / 4}}+\frac{1}{P^{1 / 4}}\right. \\
& \left.+\left(\frac{X^{\rho}}{P^{1-\tau} L^{1-\phi} H^{\rho} K^{\rho}}\right)^{1 / 2(\rho+1)}+\left(\frac{1}{P^{1-\tau} L^{1-\phi}}\right)^{1 / 2(\rho+1)}\right\}
\end{aligned}
$$

where $\rho=(1+2 k) /(4+6 k), \tau=(4+4 k) /(6+4 k)$ and $\phi=(3+2 l) /(6+4 k)$.

Sketch of the Proof. The proof is similar to that of Lemma 1.8, One starts with the Cauchy-Schwarz inequality and proceeds as in (1.6). But this time one ends up with

$$
\frac{R L}{\eta} \sum_{\substack{h_{1}, h_{2} \sim H \\ n_{1}, n_{2} \sim K \\|\Delta|<\eta}} \sum_{\substack{r \sim R \\|\Delta|<\nu}}\left|\sum_{m \sim L} e\left(2^{\gamma+\beta+1} X \Delta \frac{m^{\alpha} r^{\theta}}{L^{\alpha} P^{\theta}}\right)\right| .
$$

We next apply [18, Theorem 2] to the sums running over $r$ and $m$, and proceed similarly as in Lemma 1.8 .

For the rest of this article, we refer the reader to [16, §III.5] for some of the basic properties of the Dickman function $\rho$.

Lemma 1.10. Let $1<c<2$ be fixed and suppose $\varepsilon>0$ is fixed and small. Then for every large $x$ depending only on $c$ and $\varepsilon>0$ one has

$$
\int_{y}^{x^{c}} t^{\delta-1} \rho\left(\frac{\log t}{\log y}\right) d t \ll x \rho(c u)
$$

uniformly for $x^{c} \geqslant y \geqslant(\log x)^{c+\varepsilon}$.

Proof. Let $\delta>\kappa>0$ be a sufficiently small number to be determined. Let $f(t)=$ $t^{\delta-\kappa} \rho\left(\frac{\log t}{\log y}\right)$ defined for all $x^{c} \geqslant t \geqslant y$. It suffices to show that the relation

$$
f(t) \ll f\left(x^{c}\right)
$$


holds for all $x^{c} \geqslant t \geqslant y$, where the implied constant depends on $c$ and $\varepsilon$ but not on $y$. We may assume that $y \leqslant x^{c / 3}$, since otherwise (1.7) holds trivially. Now assume $x^{c} \geqslant t \geqslant y^{3}$; hence using [16, Lemma 8.1] and [16, Corollary 8.3], it follows that

$$
f^{\prime}(t)=t^{\delta-\kappa-1} \rho(v)\left\{\delta-\kappa-\frac{\log (v \log v)}{\log y}\left(1+O\left(\frac{\log \log v}{\log ^{2} v}\right)\right)\right\}
$$

where $v=\log t / \log y \geqslant 3$. Hence for any fixed $1<c<2$ and given $\varepsilon>0$ small, $\delta>\kappa>0$ may be chosen in such a way that $f^{\prime}(t)>0$ is satisfied at least when $y \geqslant(\log x)^{c+\varepsilon}$ for $x$ large. This proves (1.7) uniformly in $y$, when $x^{c} \geqslant t \geqslant y^{3}$. As for the case: $x^{c} \geqslant y^{3} \geqslant t \geqslant y$, (1.7) is satisfied uniformly, since $\rho$ is bounded for such values.

Lemma 1.11. Let $1<c<2$ be fixed and let $\varepsilon>0$ be a small number. Then for every large $x$ depending only on $c$ and $\varepsilon$ one has

$$
\sum_{\substack{n \leqslant x^{c} \\ P^{+}(n) \leqslant y}} \delta n^{\delta-1}=x \rho(c u)\left\{1+O\left(\frac{\log (c u+1)}{\log y}\right)\right\}
$$

uniformly for $y$ in the range $\exp \left(\left(\log \log x^{c}\right)^{5 / 3+\varepsilon}\right) \leqslant y \leqslant x^{c}$.

Proof. By partial integration, and using the facts $\rho^{\prime}(u)=0$ for $0<u<1$ and $\rho(u)=1$ for $0 \leqslant u \leqslant 1$, it follows that

$$
\int_{1}^{x^{c}} \delta t^{\delta-1} \rho\left(\frac{\log t}{\log y}\right) d t=x \rho(c u)-1-\frac{1}{\log y} \int_{y}^{x^{c}} t^{\delta-1} \rho^{\prime}\left(\frac{\log t}{\log y}\right) d t .
$$

Next, we use the inequality $\rho^{\prime}(v) \ll \log (v+1) \rho(v)$ for all $v \geqslant 0$ (see [16, Corollary 8.3]), and Lemma 1.10 to get

$$
\frac{1}{\log y} \int_{y}^{x^{c}} t^{\delta-1} \rho^{\prime}\left(\frac{\log t}{\log y}\right) d t \ll x \rho(c u) \frac{\log (c u+1)}{\log y} .
$$

By partial integration one has

$$
\sum_{\substack{n \leqslant x^{c} \\ P^{+}(n) \leqslant y}} \delta n^{\delta-1}=\int_{y^{-}}^{x^{c}} \delta t^{\delta-1} d \Psi_{1}(t, y)+y^{\delta}+O(1) .
$$

It is not hard to see that $y$ satisfies $t \geqslant y \geqslant \exp \left((\log \log t)^{5 / 3+\varepsilon}\right)$. Thus using (1.2), (1.8), (1.9) and the fact that $\rho(u)=1$ for $0 \leqslant u \leqslant 1$ (hence $\rho^{\prime}(u)=0$ except for $u=0$ and $u=1$ ), we have

$$
\int_{y^{-}}^{x^{c}} \delta t^{\delta-1} d \Psi_{1}(t, y)=x \rho(c u)\left\{1+O\left(\frac{\log (c u+1)}{\log y}\right)\right\}+\int_{y^{-}}^{x^{c}} \delta t^{\delta-1} d R(t)-y^{\delta}
$$

where $R(t) \ll t \rho(\log t / \log y) \log (\log t / \log y+1) \log ^{-1} y$. Here by partial summation it follows that the integral involving $R(t)$ is

$$
\ll \frac{x \rho(c u) \log (c u+1)}{\log y}+\frac{y^{\delta} \log (c u+1)}{\log y}+\frac{\log (c u+1)}{\log y} \int_{y}^{x^{c}} t^{\delta-1} \rho\left(\frac{\log t}{\log y}\right) d t,
$$

which is easily seen to be absorbed into the error term. Hence the result follows. 
Lemma 1.12. Let $\varepsilon>0$ be small. For $1<c<2$, and $x$ large depending only on $c$ and $\varepsilon$, one has

$$
\sum_{\substack{n \leqslant x^{c} \\ P^{+}(n) \leqslant y}} \delta n^{\delta-1}=x \rho(c u) \exp \left(O\left(c u \exp \left(-(\log c u)^{3 / 5-o(1)}\right)\right)\right)
$$

uniformly for $\left(\log x^{c}\right)^{c+\varepsilon} \leqslant y \leqslant x^{c}$.

Proof. We may assume that $y \leqslant \exp \left(\left(\log \log x^{c}\right)^{5 / 3+\varepsilon}\right)$, otherwise Lemma 1.11 implies (1.10). Using (1.3) together with partial summation, it follows that the left hand side of (1.10) is

$$
\delta x \rho(c u) \exp (R(c u))+\int_{y^{3}}^{x^{c}} \delta(1-\delta) t^{\delta-1} \rho(v) \exp (R(v)) d t+O\left(y^{3 \delta}\right)
$$

where $v=\log t / \log y$ and $R(u) \ll u \exp \left(-(\log u)^{3 / 5-\varepsilon}\right)$ for $u \geqslant 1$. Next using the lower and upper bounds of $R(u)$ and noting that $\rho(u)$ is decreasing and that $-\rho^{\prime}(v) \leqslant A \log (v+1) \rho(v)$ for all $v \geqslant 3$ and for some $A>0$; and following the proof of Lemma 1.11, by integration by parts it follows that

$$
\sum_{\substack{n \leqslant x^{c} \\ P^{+}(n) \leqslant y}} \delta n^{\delta-1} \geqslant x \rho(c u) e\left(-C u \exp \left(-(\log c u)^{3 / 5-\varepsilon}\right)\right)+O\left(y^{3 \delta}\right),
$$

for some $C>0$ and every $x$ large, and

$$
\sum_{\substack{n \leqslant x^{c} \\ P^{+}(n) \leqslant y}} \delta n^{\delta-1} \leqslant x \rho(c u) e\left(C^{\prime} u \exp \left(-(\log c u)^{3 / 5-\varepsilon}\right)\right)+O\left(y^{3 \delta} \exp \left(C^{\prime} R(c u)\right)\right),
$$

for some $C^{\prime}>0$ and every large $x$.

The asymptotic $\rho(u) \sim u^{-u(1+o(1))}$ (see [16, Theorem 8]), and the assumption $y \leqslant \exp \left(\left(\log \log x^{c}\right)^{5 / 3+\varepsilon}\right)$ now implies (1.10).

The following result will handle the ranges of small $y$ 's in Theorems 1.1 and 1.2 ,

Lemma 1.13. Assume that $(k, l)$ is an exponent pair. For $1<c<2$, let $\mathcal{I}_{c}(k, l, x, y)$ be defined as in (1.18). Then uniformly for $y$ in the range $1<y \leqslant x^{c}$, one has

$$
\sum_{\substack{1 \leqslant n \leqslant x^{c} \\ P^{+}(n) \leqslant y}} \Delta \psi(n) \ll \mathcal{I}_{c}(k, l, x, y),
$$

where the implied constant depends only on $(k, l)$, and $c$.

Proof. Let $2 \leqslant y \leqslant M \leqslant x^{c}$ be a parameter to be chosen. Then

$$
\sum_{\substack{1 \leqslant n \leqslant x^{c} \\ P^{+}(n) \leqslant y}} \Delta \psi(n)=\sum_{\substack{M<n \leqslant x^{c} \\ P^{+}(n) \leqslant y}} \Delta \psi(n)+O(M) .
$$

We first divide the interval $\left(M, x^{c}\right]$ into intervals of the form $\left(N, N^{\prime}\right]$, where $N^{\prime}=$ $\max \left\{2 N, x^{c}\right\}$. We assume $N \geqslant H_{N}>1$ and apply Lemma 1.4, on each such interval, thus end up with estimating

$$
N H_{N}^{-1}+H_{N}^{1 / 2} N^{\delta / 2}+N^{\delta-1} \max _{N<N^{\prime} \leqslant 2 N} \sum_{1 \leqslant h \leqslant H_{N}}\left|\sum_{\substack{N<n \leqslant N^{\prime} \\ P^{+}(n) \leqslant y}} e\left(h n^{\delta}\right)\right|
$$


for all $M<N \leqslant x^{c}$. With the choice of $R=M$ in Lemma 1.6 since $y \leqslant M \leqslant$ $N<n \leqslant N^{\prime}$, one has

$$
\sum_{1 \leqslant h \leqslant H_{N}}\left|\sum_{\substack{n \sim N \\ P^{+}(n) \leqslant y}} e\left(h n^{\delta}\right)\right|=\sum_{1 \leqslant h \leqslant H_{N}} \varepsilon_{h} \sum_{\substack{p \leqslant y \\ P_{M / p<u \leqslant M}}} \sum_{\substack{v \sim N / u p \\ P^{-}(u) \geqslant p \\ P^{+}(u) \leqslant y}} e\left(h p^{\delta} u^{\delta} v^{\delta}\right)
$$

for some $\varepsilon_{h}$ complex number not exceeding one in modulus. Next, we divide the ranges of $h, p, v$ and $u$ into $\ll \log y \log M \log ^{2} N \ll \log ^{4} N$ dyadic intervals to get

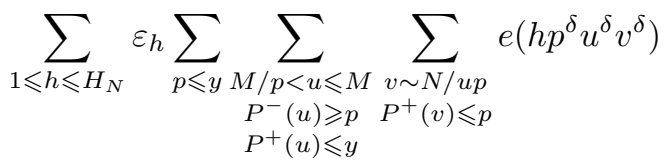

$$
\begin{aligned}
& \leqslant \sum_{\substack{1 \leqslant H \leqslant H_{N} \\
H=2^{l}}} \sum_{\substack{2 \leqslant P \leqslant y \\
P=2^{k}}} \sum_{\substack{M / 2 P \leqslant L \leqslant M \\
L=2^{r}}} \sum_{\substack{N \leqslant K \leqslant 2 N / P L \\
K=2^{j}}}\left|S^{\prime}(H, P, K, L)\right|
\end{aligned}
$$

with

$$
S^{\prime}:=S^{\prime}(H, P, K, L)=\sum_{h \sim H} \sum_{v \sim K} \sum_{\substack{p \sim P \\ P^{+}(v) \leqslant p}} \sum_{\substack{u \sim L \\ u v p \sim N}} \varepsilon_{h} b(u, p) e\left(h p^{\delta} u^{\delta} v^{\delta}\right)
$$

where $b(u, p)$ is a complex number not exceeding one in modulus. Here we note that

$$
S^{\prime}=\int_{-1 / 2}^{1 / 2} \sum_{h \sim H} \sum_{v \sim K} \sum_{u \sim L} \sum_{\substack{p \sim P \\ P^{+}(v) \leqslant p}} \varepsilon_{h} e(\alpha u p) b(u, p) e\left(h p^{\delta} u^{\delta} v^{\delta}\right) \sum_{f \sim \frac{N}{v}} e(-\alpha f) d \alpha .
$$

Using the elementary bounds (see [6, Theorem 2.1])

$$
\int_{-1 / 2}^{1 / 2} \sum_{r \in I} e(\alpha r) d \alpha \ll \int_{-1 / 2}^{1 / 2} \min \left\{Q, \frac{1}{\|\alpha\|}\right\} d \alpha \ll \log 2 Q,
$$

which hold for any interval $I \subset[Q, 2 Q)$, where $Q \geqslant 1$, it follows that

$$
S^{\prime} \log ^{-1} N \ll\left|\sum_{h \sim H} \sum_{v \sim K} a(v, h) \sum_{u \sim L} \sum_{\substack{p \sim P \\ P^{+}(v) \leqslant p}} e(\alpha u p) b(u, p) e\left(h p^{\delta} u^{\delta} v^{\delta}\right)\right|
$$

for some $\alpha \in[-1 / 2,1 / 2]$, and $a(v, h)$ at most one in modulus. We can also omit the condition $P^{+}(v) \leqslant p$ by noting

$$
\sum_{h \sim H} \sum_{v \sim K} a(v, h) \sum_{u \sim L} \sum_{\substack{p \sim P \\ P^{+}(v) \leqslant p}} e(\alpha u p) b(u, p) e\left(h p^{\delta} u^{\delta} v^{\delta}\right)
$$

$$
=\int_{-1 / 2}^{1 / 2} \sum_{h \sim H} \sum_{v \sim K} a(v, h) \sum_{u \sim L} \sum_{p \sim P} e(\alpha u p-\gamma p) b(u, p) e\left(h p^{\delta} u^{\delta} v^{\delta}\right) \sum_{\substack{f \sim P \\ P^{+}(v) \leqslant f}} e(\gamma f) d \gamma,
$$

and using the bound in (1.16). 

is

Hence, grouping the terms corresponding to $r=u p$, it follows that $S^{\prime} \log ^{-2} N$

$$
\ll \sup _{|a(v, h)| \leqslant 1} \sup _{|c(r)| \leqslant d(r)}\left|\sum_{H<h \leqslant 2 H} \sum_{K<v \leqslant 2 K} a(v, h) \sum_{P L<r \leqslant 4 P L} c(r) e\left(h r^{\delta} v^{\delta}\right)\right|,
$$

where the first supremum is taken over all coefficients that are at most one, and the second supremum is taken over all arithmetical functions that are bounded in absolute value by the ordinary divisor function. To apply Lemma 1.8 to this last exponential sum, we should estimate the mean-square of the coefficients $|c(r)|$. Thus for $R \geqslant 1$, one has

$$
\begin{aligned}
\frac{1}{R} \sum_{r \sim R}|c(r)|^{2} \leqslant 2 \sum_{r \sim R} \frac{d^{2}(r)}{r} & \leqslant 2 \prod_{p \leqslant 2 R}\left(1+\frac{4}{p}+\frac{d^{2}\left(p^{2}\right)}{p^{2}} \ldots\right) \\
& \ll \prod_{p \leqslant 2 R}\left(1+\frac{1}{p}\right)^{4} \\
& \ll \log ^{4} 2 R
\end{aligned}
$$

where we have used $d(n) \ll_{\varepsilon} n^{\varepsilon}$ for any $\varepsilon>0$ (see [16, Corollary 1.1]), and Merten's estimate (see [16, Theorem 11]).

Splitting the innermost sum into two parts and applying Lemma 1.8 on each part together with the relations $P L K \asymp N$ and $M \ll L P \ll M P$; and the uniform bound $\sum_{r \sim R}|c(r)|^{2} \ll R \log ^{4} 2 R$, it follows that

$$
\begin{aligned}
S^{\prime} \log ^{-5} N \ll H N M^{-\frac{1+k-l}{2(k+1)}}+H^{1 / 2} N^{1 / 2} & P^{1 / 2} M^{1 / 2} \\
& +H^{1 / 2} N^{1-\delta / 2}+H N^{\frac{k(\delta+1)+2}{2(k+1)}} M^{-\frac{1-l}{2(k+1)}}
\end{aligned}
$$

Summing over $H, P, K$ and $L$, multiplying by $N^{\delta-1}$ and inserting this last estimate into (1.14) and then choosing $1 \leqslant H_{N} \leqslant N$ optimally by Lemma 1.5, it follows that the right hand side of (1.14) is

$$
\begin{aligned}
\ll & \log ^{9} N\left\{N^{\delta} M^{-\frac{1+k-l}{2(k+1)}}+N^{\delta-1 / 2} y^{1 / 2} M^{1 / 2}+N^{\frac{k(\delta+1)+2}{2(k+1)}+\delta-1} M^{-\frac{1-l}{2(k+1)}}\right. \\
& \left.+N^{\frac{\delta+1}{3}}+N^{\frac{k(\delta+1)+2}{4(k+1)}+\frac{\delta}{2}} M^{-\frac{1-l}{4(k+1)}}+N^{\frac{\delta+1}{2}} M^{-\frac{1+k-l}{4(k+1)}}+N^{2 \delta / 3} y^{1 / 3} M^{1 / 3}\right\} .
\end{aligned}
$$

Summing over $M \leqslant N=2^{k} \leqslant x^{c}$, and taking into account the other term in (1.13), we see that the left hand side of (1.12) is

$$
\begin{aligned}
\ll & \log ^{10} x\left\{x^{\frac{3 k+2-c k}{2(k+1)}} M^{-\frac{1-l}{2(k+1)}}+x^{\frac{3 k+2+c(k+2)}{4(k+1)}} M^{-\frac{1-l}{4(k+1)}}+x^{\frac{1+c}{2}} M^{-\frac{1+k-l}{4(k+1)}}\right. \\
& \left.+x M^{-\frac{1+k-l}{2(k+1)}}+x^{1-c / 2} y^{1 / 2} M^{1 / 2}+x^{2 / 3} y^{1 / 3} M^{1 / 3}+M+x^{\frac{c+1}{3}}\right\}
\end{aligned}
$$


for all $y \leqslant M \leqslant x^{c}$. Here the third term dominates the fourth. We choose $y<$ $M \leqslant x^{c}$ optimally by Lemma 1.5 to see that the left hand side of (1.12) is

$$
\begin{gathered}
\ll \log ^{10} x\left\{x^{1-c / 2} y+x^{2 / 3} y^{2 / 3}+y+x^{(c+1) / 3}+x^{\frac{3 k+2-c(1+k-l)}{2(k+1)}}+x^{\frac{3 k+2+c(k+1+l)}{4(k+1)}}\right. \\
+x^{\frac{2 k+2+c(k+l+1)}{4(k+1)}}+x^{\frac{3 k+2-c k}{2 k+3-l}}+x^{\frac{3 k+2+c(k+2)}{4 k+5-l}}+x^{\frac{(1+c) 2(k+1)}{5+5 k-l}}+x^{\frac{3 k-2 l+4+c(k+2)}{4 k+7-3 l}} y^{\frac{1-l}{4 k+7-3 l}} \\
+x^{\frac{3 k+4-2 l-c k}{5+2 k-3 l}} y^{\frac{1-l}{5+2 k-3 l}}+x^{\frac{4+4 k-2 l+2 c(k+1)}{7 k+7-3 l}} y^{\frac{1+k-l}{7 k+7-3 l}}+x^{\frac{4 k+4-2 l+c(k+l+1)}{6+6 k-2 l}} y^{\frac{1+k-l}{6+6 k-2 l}} \\
\left.+x^{\frac{3 k+4-2 l+c(k+l+1)}{4 k-2 l+6}} y^{\frac{1-l}{4 k-2 l+6}}+x^{\frac{3 k-2 l+4-c(k-l+1)}{2 k-2 l+4}} y^{\frac{1-l}{2 k-2 l+4}}\right\}=\mathcal{I}_{c}(k, l, x, y) .
\end{gathered}
$$

Here it is clear that this last upper bound is worse than the trivial bound whenever $y \geqslant x^{1 / 2}$. Thus for larger values of $y$, we combine Lemma 1.13 and the next lemma.

Lemma 1.14. Suppose $1<c<2$. Let $2 \leqslant L<y \leqslant x^{c}$ be any numbers. Then

$$
\sum_{\substack{1 \leqslant n \leqslant x^{c} \\ L<P^{+}(n) \leqslant y}} \Delta \psi(n) \ll x^{\varepsilon}\left\{x^{\frac{3 c+1}{4}} L^{-1 / 4}+x^{c} L^{-1 / 2}+x^{\frac{2 c+1}{4}} y^{1 / 4}\right\}+x / \log x
$$

for any $\varepsilon>0$. Furthermore, to omit $x^{\varepsilon}$, the above upper bound may be replaced by

$$
\sum_{\substack{1 \leqslant n \leqslant x^{c} \\ L<P^{+}(n) \leqslant y}} \Delta \psi(n) \ll \log ^{8} x\left\{x^{\frac{5 c+1}{6}} L^{-1 / 6}+x^{c} L^{-1 / 3}+x^{1 / 2} y^{1 / 2}\right\}+x / \log x .
$$

The implied constant depends only on $c$ in both statements.

Proof. Splitting the sum into $\ll \log x$ dyadic sums of the form $\left(N, N^{\prime}\right]$, where $N^{\prime}=\max \left(2 N, x^{c}\right)$, we apply Lemma 1.4. Thus for $N \geqslant L \geqslant 2$, we estimate

$$
N H_{N}^{-1}+H_{N}^{1 / 2} N^{\delta / 2}+N^{\delta-1} \max _{N<N^{\prime} \leqslant 2 N} \sum_{1 \leqslant h \leqslant H_{N}}\left|\sum_{\substack{N<n \leqslant N^{\prime} \\ L<P^{+}(n) \leqslant y}} e\left(h n^{\delta}\right)\right|
$$

where $1 \leqslant H_{N} \leqslant N$ is to be chosen. To this end we set

$$
\begin{aligned}
S(N) & =\sum_{1 \leqslant h \leqslant H_{N}}\left|\sum_{\substack{N<n \leqslant N^{\prime} \\
L<P^{+}(n) \leqslant y}} e\left(h n^{\delta}\right)\right| \\
& =\sum_{1 \leqslant h \leqslant H_{N}} \varepsilon_{h} \sum_{\substack { L<p \leqslant y \\
\begin{subarray}{c}{N / p<n \leqslant N^{\prime} / p \\
P^{+}(n) \leqslant p{ L < p \leqslant y \\
\begin{subarray} { c } { N / p < n \leqslant N ^ { \prime } / p \\
P ^ { + } ( n ) \leqslant p } }\end{subarray}} e\left(h p^{\delta} n^{\delta}\right)
\end{aligned}
$$

for some $\varepsilon_{h}$ complex number with modulus at most one. We next divide all the ranges of $h, p$ and $n$ into dyadic intervals:

$$
S(N) \leqslant \sum_{\substack{H \leqslant H_{N} \\ H=2^{k}}} \sum_{\substack{L<P \leqslant y \\ P=2^{s}}} \sum_{\substack{N / 2 P X \leqslant 2 N^{\prime} / P \\ X=2^{m}}}|S(X, P, H)|
$$


where

$$
S=S(X, P, H)=\sum_{h \sim H} \sum_{n \sim X} \sum_{\substack{p \sim P \\ n p \sim N \\ P^{+}(n) \leqslant p}} \varepsilon_{h} e\left(h n^{\delta} p^{\delta}\right) .
$$

The conditions $P^{+}(n) \leqslant p$ and $n p \sim N$ may be omitted as we did in (1.17) yielding

$$
S \ll \log N \sup _{\alpha \in[-1 / 2,1 / 2]}\left|\sum_{H<h \leqslant 2 H} \sum_{X<n \leqslant 2 X} \sum_{P<p \leqslant 2 P} a(n, h) e(\alpha p) e\left(h n^{\delta} p^{\delta}\right)\right|
$$

for some complex number $a(n, h)$ with modulus at most one. Applying Lemma 1.7 together with the relation $P X \asymp N$ yields

$$
S N^{-\varepsilon} \ll H N^{\frac{3+\delta}{4}} P^{-1 / 4}+H^{3 / 4} N^{3 / 4} P^{1 / 4}+H N P^{-1 / 2}+H^{1 / 2} N^{1-\delta / 2},
$$

for any $\varepsilon>0$. Summing over $H, X, P$, and choosing $H_{N}=N^{1-\delta+2 \varepsilon}$, inserting this last estimate into (1.21) and finally summing over $N$, we arrive at (1.19) with $\varepsilon$ replaced by $4 \varepsilon$. As for (1.20), we apply Lemma 1.8 together with the choice $(k, l)=(1 / 2,1 / 2)$ and proceed similarly, but this time with $H_{N}=N^{1-\delta} \log ^{2} N$.

\subsection{Proof of Theorems 1.1 and $\mathbf{1 . 2}$.}

Proof of Theorem 1.1. By Lemmas 1.3 and 1.11, it suffices to show that, given $1<c<24979 / 20803$, one has

$$
\sum_{\substack{1 \leqslant n \leqslant x^{c} \\ P^{+}(n) \leqslant y}} \Delta \psi(n)=O\left(\frac{x \rho(c u) \log (u+1)}{\log y}\right)
$$

uniformly for the range of $y$ in Theorem 1.1. Using the asymptotic $\rho(u) \sim u^{-u(1+o(1))}$ (see [16, Theorem 8]), it suffices to show that the left hand side of (1.22) is « $x / \log x$ whenever $u$ is uniformly bounded, and is $\ll x^{1-\varepsilon}$ whenever $u$ increases indefinitely subject to $y \geqslant \exp \left(\left(\log \log x^{c}\right)^{5 / 3+\varepsilon}\right)$.

Bearing this in mind, in Lemma 1.13, we first take

$$
(k, l)=B(1 / 162,359 / 378+\varepsilon)=(85 / 189+\varepsilon, 41 / 81) .
$$

Here for the exponent pair $(1 / 162,359 / 378+\varepsilon)$ the reader is referred to $[8$, Theorem $2]$. Examining the worst scenerio in the expression of $\mathcal{I}_{c}(k, l, x, y)$ (which in fact comes from the eleventh term) yields that for any fixed $1<c<1669 / 1389$, there is $\kappa>0$ such that for any $0<\eta<\kappa$,

$$
\sum_{\substack{1 \leqslant n \leqslant x^{c} \\ P^{+}(n) \leqslant y}} \Delta \psi(n) \ll \mathcal{I}_{c}(k, l, x, y)=O\left(\frac{x \rho(c u) \log (u+1)}{\log y}\right)
$$

holds uniformly for $\exp \left((\log \log x)^{5 / 3+\varepsilon}\right) \leqslant y \leqslant \min \left\{x^{\frac{1669-1389 c}{280}-\eta}, x^{1 / 2-\eta}\right\}$, in the case that $x$ is large.

If $2509 / 2229 \leqslant c<1669 / 1389$, then the first term in the minimum is less than the second term, giving the second range in (1.4). 
To obtain the first range in (1.4), we may assume that $y>x^{3(c-1)+\varepsilon}$, otherwise the range $\exp \left((\log \log x)^{5 / 3+\varepsilon}\right) \leqslant y \leqslant x^{3(c-1)+\varepsilon}$ lies in the range of validity of the estimate in (1.23) for $1<c<2509 / 2229$. Hence

$$
\begin{aligned}
& \sum_{\substack{1 \leqslant n \leqslant x^{c} \\
P^{+}(n) \leqslant y}} \Delta \psi(n)=\sum_{\substack{1 \leqslant n \leqslant x^{c} \\
P^{+}(n) \leqslant x^{3(c-1)+\varepsilon}}} \Delta \psi(n)+\sum_{\substack{1 \leqslant n \leqslant x^{c} \\
x^{3(c-1)+\varepsilon}<P^{+}(n) \leqslant y}} \Delta \psi(n) \\
& =\sum_{\substack{1 \leqslant n \leqslant x^{c} \\
x^{3(c-1)+\varepsilon}<P^{+}(n) \leqslant y}} \Delta \psi(n)+O\left(\frac{x}{\log x}\right) .
\end{aligned}
$$

Also by Lemma 1.14, the first sum on the right hand side of 1.24 is $\ll x / \log x$; provided that $x^{3(c-1)+\varepsilon}<y \leqslant x^{3-2 c-\varepsilon}$. Thus for a fixed $1<c<2509 / 2229$, we have shown that

$$
\sum_{\substack{1 \leqslant n \leqslant x^{c} \\ P^{+}(n) \leqslant y}} \Delta \psi(n)=O\left(\frac{x \rho(c u) \log (u+1)}{\log y}\right)
$$

uniformly for $\exp \left((\log \log x)^{5 / 3+\varepsilon}\right) \leqslant y \leqslant x^{3-2 c-\varepsilon}$.

Finally, to extend the validity of the estimate in (1.25) to $\exp \left((\log \log x)^{5 / 3+\varepsilon}\right) \leqslant$ $y \leqslant x / \log ^{18} x$, we follow the splitting argument in $(1.24)$; but this time we split the sum at $x^{3-2 c-\varepsilon}$ and use the estimate in (1.20).

Proof of Theorem 1.2. By Lemmata 1.12 and 1.3, it suffices to show that

$$
\sum_{\substack{1 \leqslant n \leqslant x^{c} \\ P^{+}(n) \leqslant y}} \Delta \psi(n)=o\left(x \rho(c u) \exp \left(O\left(c u \exp \left(-(\log c u)^{3 / 5-o(1)}\right)\right)\right)\right)
$$

uniformly for $\exp \left(\left(\log \log x^{c}\right)^{5 / 3+\varepsilon}\right) \geqslant y \geqslant \log ^{\frac{4128 c}{1669-1389 c}+\varepsilon} x$, for a fixed $1<c<$ 1669/1389, otherwise we may use Theorem 1.1.

Using the same exponent pair $(k, l)=(85 / 189+\varepsilon, 41 / 81)$, one has

$$
\sum_{\substack{1 \leqslant n \leqslant x^{c} \\ P^{+}(n) \leqslant y}} \Delta \psi(n) \ll \mathcal{I}(k, l, x, y) \ll_{\kappa} x^{\frac{1389 c+2459}{4128}+\kappa}
$$

for every $\kappa>0$ and $x$ large. Using now the asymptotic $\rho(u) \sim \frac{1}{u^{u(1+o(1))}}$, we see that (1.26) holds uniformly in $y$ for the asserted range.

\section{REFERENCES}

[1] Yıldırım Akbal and Ahmet M. Güloğlu, Waring's problem with Piatetski-Shapiro numbers, Mathematika 62 (2016), no. 2, 524-550, DOI 10.1112/S0025579315000340. MR3521340

[2] R. C. Baker, The square-free divisor problem, Quart. J. Math. Oxford Ser. (2) 45 (1994), no. 179, 269-277, DOI 10.1093/qmath/45.3.269. MR.1295577

[3] Roger C. Baker, William D. Banks, Jörg Brüdern, Igor E. Shparlinski, and Andreas J. Weingartner, Piatetski-Shapiro sequences, Acta Arith. 157 (2013), no. 1, 37-68, DOI 10.4064/aa157-1-3. MR3005098

[4] Cécile Dartyge, Greg Martin, and Gérald Tenenbaum, Polynomial values free of large prime factors, Period. Math. Hungar. 43 (2001), no. 1-2, 111-119, DOI 10.1023/A:1015237700066. MR:1830570

[5] Étienne Fouvry and Henryk Iwaniec, Exponential sums with monomials, J. Number Theory 33 (1989), no. 3, 311-333, DOI 10.1016/0022-314X(89)90067-X. MR1027058 
[6] S. W. Graham and G. Kolesnik, van der Corput's method of exponential sums, London Mathematical Society Lecture Note Series, vol. 126, Cambridge University Press, Cambridge, 1991. MR 1145488

[7] Andrew Granville, Smooth numbers: computational number theory and beyond, Algorithmic number theory: lattices, number fields, curves and cryptography, Math. Sci. Res. Inst. Publ., vol. 44, Cambridge Univ. Press, Cambridge, 2008, pp. 267-323. MR2467549

[8] D. R. Heath-Brown, A New k-th Derivative Estimate for Exponential Sums via Vinogradov's Mean Value, arXiv:1601.04493

[9] D. R. Heath-Brown, The Pjateckǐ̌-S̆apiro prime number theorem, J. Number Theory 16 (1983), no. 2, 242-266, DOI 10.1016/0022-314X(83)90044-6. MR698168

[10] Adolf Hildebrand, On the number of positive integers $\leq x$ and free of prime factors $>y$, J. Number Theory 22 (1986), no. 3, 289-307, DOI 10.1016/0022-314X(86)90013-2. MR831874

[11] Adolf Hildebrand and Gérald Tenenbaum, Integers without large prime factors, J. Théor. Nombres Bordeaux 5 (1993), no. 2, 411-484. MR.1265913

[12] Greg Martin, An asymptotic formula for the number of smooth values of a polynomial, J. Number Theory 93 (2002), no. 2, 108-182, DOI 10.1006/jnth.2001.2722. MR.1899301

[13] I. I. Pyateckiı̌-Šapiro, On the distribution of prime numbers in sequences of the form $[f(n)]$ (Russian), Mat. Sbornik N.S. 33(75) (1953), 559-566. MR0059302

[14] Joël Rivat and Patrick Sargos, Nombres premiers de la forme $\left\lfloor n^{c}\right\rfloor$ (French, with English summary), Canad. J. Math. 53 (2001), no. 2, 414-433, DOI 10.4153/CJM-2001-017-0. MR.1820915

[15] O. Robert and P. Sargos, Three-dimensional exponential sums with monomials, J. Reine Angew. Math. 591 (2006), 1-20, DOI 10.1515/CRELLE.2006.012. MR2212877

[16] Gérald Tenenbaum, Introduction to analytic and probabilistic number theory, Cambridge Studies in Advanced Mathematics, vol. 46, Cambridge University Press, Cambridge, 1995. Translated from the second French edition (1995) by C. B. Thomas. MR1342300

[17] R. C. Vaughan, A new iterative method in Waring's problem, Acta Math. 162 (1989), no. 1-2, 1-71, DOI 10.1007/BF02392834. MR 981199

[18] Jie Wu, Double exponential sums and some applications, Monatsh. Math. 128 (1999), no. 3, 255-262, DOI 10.1007/s006050050062. MR.1719419

Department of Mathematics, Bilkent University, 06800 Bilkent, Ankara, Turkey

E-mail address: yildirim.akbal@bilkent.edu.tr, yildirim.akbal@gmail.com 